\title{
Onset of Resistive Interchange Mode in the Large Helical Device
}

\author{
Satoru SAKAKIBARA, Kiyomasa WATANABE, Hiroshi YAMADA, Yoshiro NARUSHIMA, \\ Taiki YAMAGUCHI ${ }^{1)}$, Kazuo TOI, Satoshi OHDACHI, Kenji TANAKA, Tokihiko TOKUZAWA, \\ Kazumichi NARIHARA, Kazuo KAWAHATA, Akio KOMORI and LHD Experimental Group \\ National Institute for Fusion Science, Toki 509-5292, Japan \\ ${ }^{1)}$ The Graduate University for Advanced Studies, Toki 509-5292, Japan
}

(Received 31 May 2006 / Accepted 28 August 2006)

\begin{abstract}
The relationship between magnetohydrodynamic (MHD) modes and the linear stability boundary has been investigated by controlling the pressure gradient in the Large Helical Device. Edge plasma pressure was varied by inserting a pump limiter into the plasma, and the onsets of MHD modes were determined. A quantitative comparison between mode onsets and a linear stability index of the resistive interchange mode was performed under the condition of a specific magnetic Reynolds number. The experimental results are consistent within a factor of 2 with a rough estimation of the stability boundary of a resistive low- $n$ mode.
\end{abstract}

(c) 2006 The Japan Society of Plasma Science and Nuclear Fusion Research

Keywords: MHD stability, LHD, movable limiter, resistive interchange mode, magnetic Reynolds number

DOI: $10.1585 /$ pfr.1.049

Stable maintenance of a steep pressure gradient in a plasma edge with the intention of improving confinement is a prevalent issue in magnetic confinement systems, this being deeply related to the characteristics of magnetohydrodynamic (MHD) instabilities. In tokamaks, edge localized modes (ELMs) excited around the edge pedestal lead to the erosion of the divertor plate and the first wall. For example, the acceptable ELM size in the International Thermonuclear Experimental Reactor (ITER) is limited by the damage to the divertor material [1]. Active control techniques are being investigated to avoid or to suppress ELMs entirely [2]. Recent theory suggests that ELMs are driven by peeling-ballooning modes, and an experimental verification through a comparison with an excellent MHD stability code has been performed in various machines [3] and their qualitative consistencies have been obtained.

In heliotron configurations, pressure-driven interchange modes are likely to easily become destabilized even in low- $\beta$ plasma because of the existence of magnetic hill, especially, in the edge region. In the Large Helical Device (LHD), core MHD activities, which affect the plasma profile in the medium- $\beta$ range at less than $2.5 \%$, are completely stabilized by the spontaneous formation of a magnetic well due to finite- $\beta$ effects [4]. On the other hand, edge resonant modes with low- $m$ and $n$ are enhanced when $\beta$ is increased, and they sometimes limit the increment of $\beta[5,6]$. Also, an interesting phenomenon in which the dominant mode changes from that excited in the inner region to the outer one when $\beta$ increases has been observed in the high- $\beta$ regime $[7,8]$.

The phenomenological understanding of global MHD activities in heliotron configurations and their effects

author'se-mail: sakakis@lhd.nifs.ac.jp on plasma confinements has been steadily progressing through various experiments. The significance of the linear stability boundary should be clarified in regard to the design of a helical reactor, and it is important to experimentally determine the threshold of MHD instabilities limiting plasma confinement. From this point of view, systematic comparison between observed local pressure gradient and the linear growth rate of ideal low- $n$ instability has been performed in order to clarify the role of the predicted ideal linear theory in helical systems [9].

In the present paper we report the experimental results of changes of MHD activities by using a movable limiter to control edge pressure gradients directly. This series of experiments has three kinds of advantages on MHD study. First, they enable us to create detailed reconstructions of equilibria with clear plasma boundaries, which can provide accurate stability calculations. The LHD configuration has an ergodic layer outside the last closed flux surface, which makes the accuracy of the calculation of edge stability unclear. Second, the movable limiter can directly control the edge pressure and its gradient that destabilizes the pressure-driven mode. This is also useful for confirming if excited modes are the resonant modes. Third, it is possible to identify the onset of MHD instability in steady state plasmas. Previous experiments have shown that peripheral MHD modes are observed even in low- $\beta$ plasmas and that they appear in the transitional state of plasmas. This makes it relatively difficult to identify mode-onset. The present article primarily describes the relationship between the onsets of the observed MHD modes and the linear stability boundary.

The LHD is a heliotron-type device comprised of a pair of continuous helical coils and three pairs of poloidal 
coils, these coils being superconductive. The magnetic axis position, $R_{\mathrm{ax}}$, which characterizes transport and stability, can be changed from 3.4 to $4.1 \mathrm{~m}$ by controlling the poloidal coil currents. The $R_{\mathrm{ax}}$ of $3.6 \mathrm{~m}$ and toroidal magnetic field at $R_{\mathrm{ax}}, B_{\mathrm{t}}$, of $0.75 \mathrm{~T}$ were selected in the limiter experiments. The plasma aspect-ratio, $A_{\mathrm{p}}$, is set at 5.8, which is the so-called standard configuration of the LHD. This configuration is well used in high- $\beta$ experiments because the best energy confinement has been obtained in the presently available operation region, providing much experimental data in the extended $\beta$ range [6]. Figure 1 shows a schematic view of the limiter configuration. The water-cooled limiter is made of mainly stainless steel and is inserted into the plasma. It has a complex shape optimized for Local Island Divertor (LID) configuration [10]. In this configuration, the limiter is set at the inside O-point of $m / n=1 / 1$ magnetic island produced by external perturbation coils, and the particles attaching the leading edge of the limiter are exhausted to the pumping duct. Here $m$ and $n$ are poloidal and toroidal mode number, respectively. The MHD characteristics observed in the LID configuration are shown in Ref. [8]. In the experiments performed here, the limiter was set at the specific location in the configuration without the $m / n=1 / 1$ perturbation field. The vacuum pumping by the duct shown in Fig. 1 was then performed during all the discharges. For the sake of convenience, the limiter position, $R_{\mathrm{h}}$, which is the radial position of the limiter tip at $z=0.33 \mathrm{~m}$, is used. The limiter was inserted up to $R_{\mathrm{h}}=4.02 \mathrm{~m}$, which almost exactly corresponds to that just inside $\iota / 2 \pi=1$ resonant surface.

The volume-averaged beta value, $\left\langle\beta_{\mathrm{dia}}\right\rangle$ is estimated by the diamagnetic measurements and defined as $4 \mu_{0} / 3$. $W_{\text {dia }} /\left(B_{\text {av }}{ }^{2} V_{\mathrm{p}}\right)$, where $W_{\text {dia }}$ is the diamagnetic energy. $B_{\text {av }}$ and $V_{\mathrm{p}}$ are the averaged toroidal magnetic field inside the plasma boundary and the plasma volume, respectively. The plasma boundary is defined by the profile measurements. The electron temperature profile is measured using

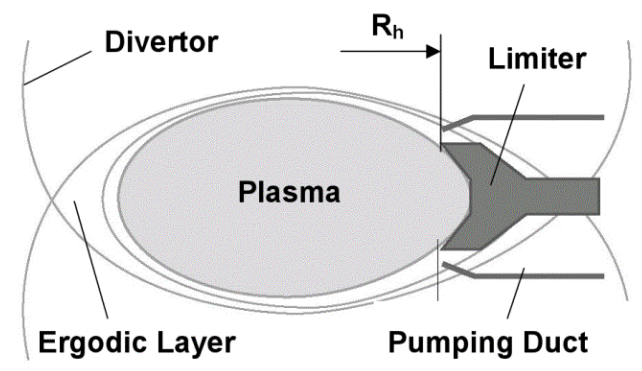

Fig. 1 Schematic view of the limiter configuration. The $R_{\mathrm{h}}$ is the radial position of the limiter tip at $z=0.33 \mathrm{~m}$ and used as the limiter position. a Thomson scattering system. The line-averaged electron density and its profile are estimated by measurement using an FIR interferometer. The electron pressure profile and the local gradient are estimated by both profile measurements.

Figure 2 shows changes of the central beta, $\beta_{0},\left\langle\beta_{\mathrm{dia}}\right\rangle$ and the line-averaged electron density, $\bar{n}_{\mathrm{e}}$, as a function of $R_{\mathrm{h}}$. The target plasmas are produced and maintained by the tangentially balanced injection of two neutral beams. Total port-through power is about $4 \mathrm{MW}$ and the electron density is sustained by a Hydrogen gas-puff. The limiter was inserted every $0.02 \mathrm{~m}$ from $R_{\mathrm{h}}=4.4 \mathrm{~m}$ into such background plasmas. In the case of $R_{\mathrm{h}}=4.5 \mathrm{~m}$ (hatched area), which corresponds to the reference plasma without the limiter insertion, the $\beta_{0}$ and $\left\langle\beta_{\text {dia }}\right\rangle$ are about $3 \%$ and $1.3 \%$, respectively. The $\beta_{0}$ increases with the insertion of the limiter and starts to decrease when $R_{\mathrm{h}}$ approaches $4.34 \mathrm{~m}$, while $\left\langle\beta_{\text {dia }}\right\rangle$ is almost constant when $R_{\mathrm{h}}>4.3 \mathrm{~m}$ and decreases with reduction of $R_{\mathrm{h}}$. The $\bar{n}_{\mathrm{e}}$ gradually decreases from $1.8 \times 10^{19} \mathrm{~m}^{-3}$ with the insertion of the limiter.

Changes of the plasma boundaries defined by different conditions and radial positions of the rational surfaces as a function of $R_{\mathrm{h}}$ are shown in Fig. 3. Clear identification of the plasma boundary experimentally is difficult because of the lack of measurements at the edge, so two kinds of plasma boundaries are estimated here. One is defined by the extrapolation of the observed $T_{\mathrm{e}}$ profile, while the other is determined by the location where the plasma has the ratio of the specific amount of the electron stored energy to total one. The locations of resonant surfaces, which are calculated by the 3-D MHD equilibrium code VMEC [11], are nearly constant at any limiter position. Low- $n$ resonant edge surfaces with $m / n=2 / 3,3 / 4$, and $4 / 5$ go beyond the plasma boundary when $R_{\mathrm{h}}<4.13 \mathrm{~m}$, and the $\iota / 2 \pi=1$ surface becomes the plasma boundary at $R_{\mathrm{h}}=4.02 \mathrm{~m}$. Thus,

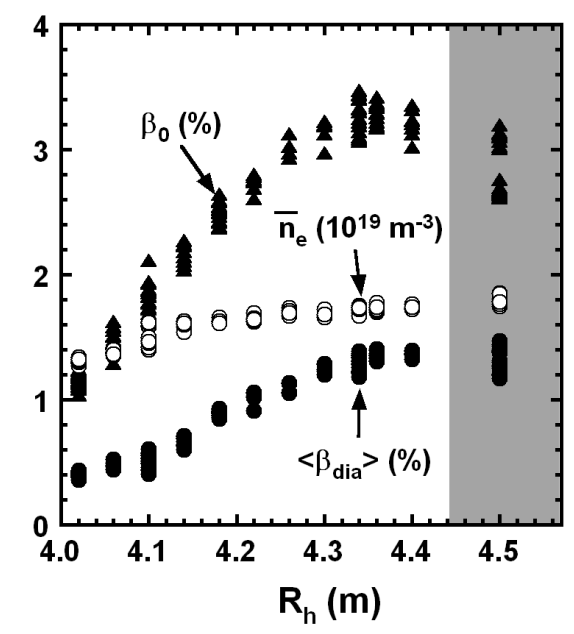

Fig. 2 Changes of $\beta_{0}$ (closed triangle), $\bar{n}_{\mathrm{e}}$ (open circle), and $\left\langle\beta_{\mathrm{dia}}\right\rangle$ (closed circle) as a function of $R_{\mathrm{h}}$. The hatched area is the reference plasma without the limiter. 


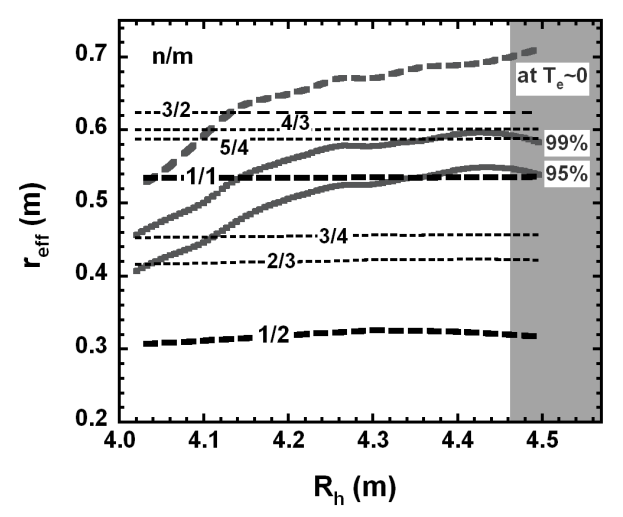

Fig. 3 Changes of specific plasma boundaries and radial positions of resonant surfaces $(n / m=1 / 2,2 / 3,3 / 4,1 / 1,5 / 4$, $4 / 3$, and 3/2) as a function of $R_{\mathrm{h}}$. The plasma boundaries were decreased with the insertion of the limiter.

the pressures at the $\iota / 2 \pi \geq 1$ resonant surfaces could be completely reduced in the experiments.

Figure 4 shows changes of effective $\beta$ gradients, $R_{\mathrm{c}} \mathrm{d} \beta / \mathrm{d} r_{\text {eff }}$, around the $m / n=1 / 1,3 / 4$, and $2 / 3$ rational surfaces and the amplitudes of their modes as a function of $R_{\mathrm{h}}$. The $r_{\mathrm{eff}}$ and $R_{\mathrm{c}}$ are the effective plasma radius and the major radius, respectively, and $R_{\mathrm{c}}$ is $3.9 \mathrm{~m}$. The $\beta$ is estimated by using the local plasma pressure and averaged toroidal field. These data were obtained during steadystate plasmas with $\mathrm{d} W_{\mathrm{p}} / \mathrm{d} t \sim 0$, where $W_{\mathrm{p}}$ is the stored energy. The $\beta$ gradient at the $\iota / 2 \pi=4 / 3$ surface decreases with insertion of the limiter, and it becomes nearly zero when $R_{\mathrm{h}}$ reaches $4.1 \mathrm{~m}$. The $\beta$ gradient at the $\iota / 2 \pi=1$ surface, keeping a relatively higher value than the $\iota / 2 \pi=4 / 3$ case, decreases with the decrease in $R_{\mathrm{h}}$. It is almost zero when $R_{\mathrm{h}}$ is $4.02 \mathrm{~m}$, which corresponds to the disappearance of the resonance as shown in Fig. 3. Unfortunately, the information on the $\iota / 2 \pi=3 / 2$ surface could not be obtained except for the case of $R_{\mathrm{h}} \leq 4.14 \mathrm{~m}$ because the plasma boundary is defined as the location just inside the $\iota / 2 \pi=3 / 2$ resonance. The lack of the edge measurement and the existence of the ergodic layer make accurate equilibrium reconstruction difficult. The equilibrium calculation by VMEC is valid only on the assumption that the magnetic surfaces are completely nested.

The mode amplitudes shown in Fig. 4 (b) are defined as a root mean square of magnetic fluctuations during a period of $10 \mathrm{~ms}$, and each datum is synchronized with the profile measurements. While the $m / n=2 / 3$ and $3 / 4$ modes are observed when $R_{\mathrm{h}} \geq 4.34 \mathrm{~m}$, they are completely suppressed when $R_{\mathrm{h}}<4.34 \mathrm{~m}$ in spite of the existing finite $\beta$ gradient. The $m / n=1 / 1$ mode is enhanced by the limiter insertion until $R_{\mathrm{h}}$ reaches $4.34 \mathrm{~m}$, and their amplitudes gradually decrease when $R_{\mathrm{h}}<4.34 \mathrm{~m}$. When $R_{\mathrm{h}}$ approaches $4.1 \mathrm{~m}$, the modes become intermittent and their amplitudes are sufficiently small.

The results of the linear stability analyses on the resis-

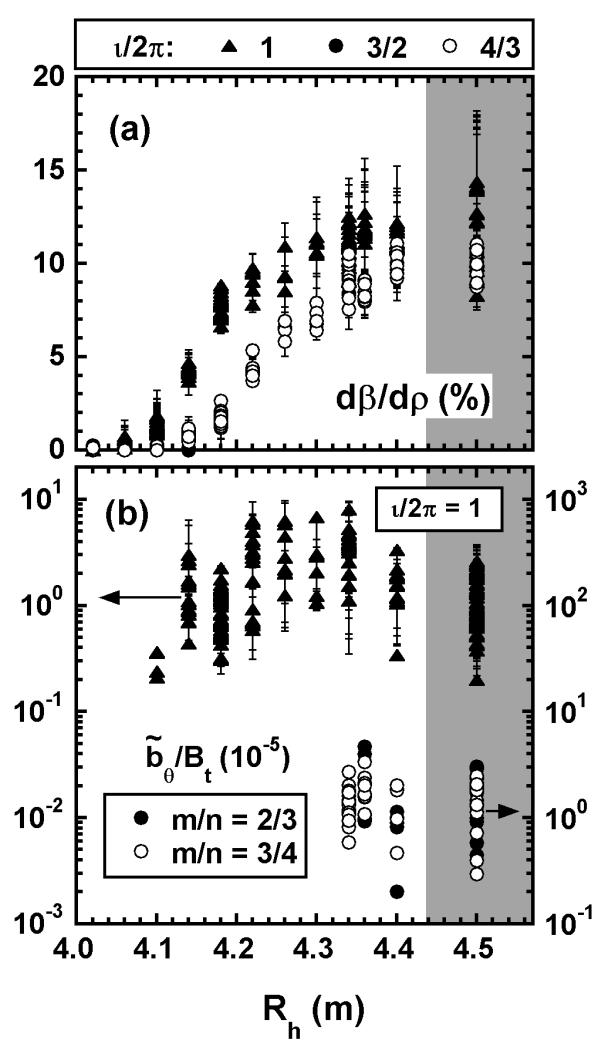

Fig. 4 Changes of (a) effective $\beta$ gradients at $\iota / 2 \pi=1$ (closed triangle), $3 / 2$ (closed circle), and 4/3 (open circle) resonant surfaces and (b) amplitudes of $\iota / 2 \pi=1$ resonant modes and $m / n=2 / 3$ and $3 / 4$ modes as a function of $R_{\mathrm{h}}$. The $\beta$ gradient at $\iota / 2 \pi=3 / 2$ resonant surface was not measured.

tive interchange mode and changes of magnetic Reynolds numbers, $S$, at $\iota / 2 \pi=4 / 3$ and 1 resonances are shown in Fig. 5. The "stable" regime shown in the figure corresponds to that in which the modes were not observed. The ideal interchange mode is theoretically stable in the edge region due to the magnetic shear effect. The $D_{\mathrm{R}}$ can be used as an accurate index of the stability boundary of the resistive mode [12]. A positive $D_{\mathrm{R}}$ indicates that the resistive mode is unstable, and edge MHD modes are linearly unstable magentic hill configurations in a series of experiments. When the $\iota / 2 \pi=4 / 3$ and 1 resonant modes disappear or are sufficiently suppressed, both $D_{\mathrm{R}}$ 's have the same value of $0.08 \sim 0.1$. The $S$ parameter, which concerns the linear growth rate of the resistive modes, continues to decrease with the limiter insertion because of the reduction of $T_{\mathrm{e}}$. Both thresholds of the $D_{\mathrm{R}}$ are obtained in the plasmas with $S$ of $5 \times 10^{5} \sim 10^{6}$. The relationships between the amplitudes of $m / n=1 / 1$ and 3/4 modes, $S$ and $D_{\mathrm{R}}$ are summarized in Fig. 6. The experiments were performed in the range with $S=10^{3} \sim 10^{7}$ and $D_{\mathrm{R}}=0 \sim 0.3$. In the case of the same $D_{\mathrm{R}}$, the amplitudes of the modes in the low- $S$ case are larger than those of the high- $S$ case. According to the linear theory of the resistive interchange 


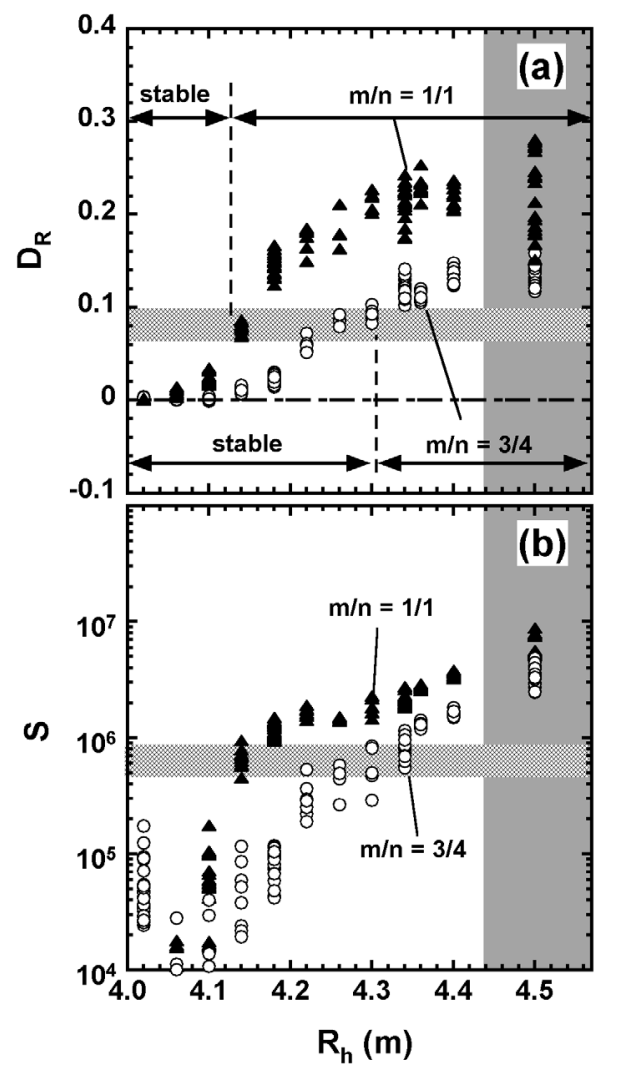

Fig. 5 Changes of (a) $D_{\mathrm{R}}$ and (b) $S$ around the $m / n=1 / 1$ and $3 / 4$ resonant surfaces as a function of $R_{\mathrm{h}}$. The "stable" region corresponds to the disappearance of the mode.

mode, the linear growth rate is proportional to $S^{-1 / 3}$ in the region with $S \geq 10^{6}$, and therefore the observation results are qualitatively consistent with the theory, if the growth rate is related with the radial extension of the mode. A detailed comparison of numerical predictions and experimental results regarding the mode's spatial structure will be the subject of future study.

The stability analysis focused on quasi-steady state plasma with different $R_{\mathrm{h}}$. A direct comparison between the observed mode and the linear calculation is not valid because the mode is predicted to already be in a nonlinear saturation state. Therefore, we focus on the plasma parameters without the appearance of the mode only, and this analysis corresponds to that just before the growth of the mode in one discharge. Also, note that the observed mode is assumed to be a single one. Here, the $m / n=1 / 1$ and $3 / 4$ modes were observed in a frequency range of less than $10 \mathrm{kHz}$, whereas their sideband components, that is, $(m \pm 1) / n$, were not observed in a frequency range of up to $50 \mathrm{kHz}$.

The significance of linear stability parameters such as $D_{\text {I }}$ and $D_{\mathrm{R}}$ for the optimization of the magnetic configuration has been pointed out by previous theoretical works, and a comparison between theoretical and experimental results has been performed using several small and middle-

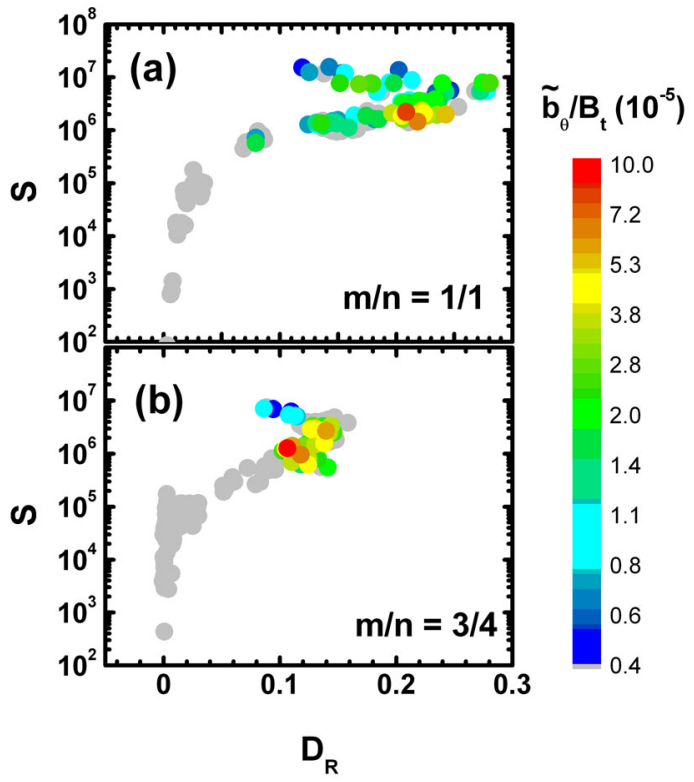

Fig. 6 Amplitudes and onsets of (a) $m / n=1 / 1$ and (b) $m / n=$ $3 / 4$ modes in the $S$ and $D_{\mathrm{R}}$ diagram.

size machines [13], where $D_{\mathrm{I}}$ is the index of the ideal stability boundary. The Compact Helical System (CHS) and LHD experiments have indicated that operation in the Mercier unstable region with no strong instability is possible, while it has been found that the MHD modes almost appear in the unstable regions of the ideal and resistive interchange modes [4]. On the contrary, the amplitudes of the modes observed in the CHS are much higher than those in the case of the LHD, which is considered to be due to the difference of the magnetic Reynolds number [14]. The limiter experiments are expected to clarify the role of the linear stability parameters, which is related to the growth rate, the radial structure of the mode and so on, in the excitation of the modes through the relative accurate stability calculation. Also, it enables us the control of $S$ parameters.

Here we focused the onset parameters of the edge modes on the $S$ and $D_{\mathrm{R}}$ planes. Experimental data show that the edge MHD modes appear when $D_{\mathrm{R}}$ is about $0.08 \sim$ 0.1 in the plasmas with $S \sim 10^{6}$. According to Ref. [15], the low- $n$ resistive modes have a significant growth rate when $D_{\mathrm{R}}$ is $0.15 \sim 0.20$ in the plasmas with $S \geq 10^{6}$, which is consistent with the experimental results within a factor of two. Note that a series of calculations was done by using the 2D MHD stability code. Although such approximate calculations may be valid for a configuration with a large-aspect ratio such as those of the Heliotron-E with $A_{\mathrm{p}} \sim 10.3$, the low-aspect-ratio configurations such as those of the LHD with $A_{\mathrm{p}} \sim 5.8$ and the CHS with $A_{\mathrm{p}} \sim 5$ require the $3 \mathrm{D}$ calculation on the low- $n$ mode for a strict comparison with the experimental results.

Regarding the amplitudes of the observed modes, which may correspond to the state of the mode saturation 
due to non-linear effects, the complex behavior of the amplitude of the $m / n=1 / 1$ mode had been determined with a reduction of $D_{\mathrm{R}}$ and $S$, as shown in Fig. 6. Generally, a reduction of the $S$ parameter corresponds to the increment of the growth rate, while a decrease in $D_{\mathrm{R}}$ leads to stabilize the mode [15]. This suggests that mode activity can be characterized not only by the pressure gradient and configuration, but that the $S$ parameter may also be one of the key issues regarding the saturation level of the modes. In recent experiments, a clear dependence of the mode amplitudes on $S$ parameter has been revealed [16]. Reference [9] shows that the mode structure is extended in the radial direction with the increase in the growth rate, although that occurs only in the case of the ideal low- $n$ mode. Thus, the relation between the radial structure of the resistive low- $n$ mode and the measurements should be clarified.

In summary, MHD activities in the limiter plasma have been investigated for clarifying the validity of the linear stability boundary. It is verified that all the observed modes are resonant ones, and their appearances are qualitatively consistent with the prediction by the linear theory regarding resistive interchange modes. The linear stability parameters at the onsets of the edge modes are relatively higher than those on the assumption of the high- $n$ mode, and they are consistent with the rough prediction of the low- $n$ resistive modes within a factor of 2 . Also, it has been revealed that the saturation levels of mode amplitudes depend on the magnetic Reynolds number in addition to the pressure gradient and the magnetic configuration.

[1] G. Federici et al., Plasma Phys. Control. Fusion 45, 1523 (2003).

[2] L.D. Horton et al., Plasma Phys. Control. Fusion 46, B511 (2004).

[3] P.B. Snyder et al., Nucl. Fusion 44, 320 (2004).

[4] S. Sakakibara et al., Plasma Phys. Control Fusion 44, A217 (2002).

[5] K. Toi et al., Nucl. Fusion 44, 217 (2004).

[6] K.Y. Watanabe et al., Fusion Sci. Tech. 46, 24 (2004).

[7] S. Sakakibara et al., Proc. 31st EPS conference on Plasma Phys. London, 28 June - 2 July 2004 ECA Vol.28G, O-4.01 (2004).

[8] A. Komori et al., Phys. Plasmas 12, 056122 (2005).

[9] K.Y. Watanabe et al., Nucl. Fusion. 45, 1247 (2005).

[10] A. Komori et al., J. Nucl. Mater. 241-243, 967 (1997).

[11] S.P. Hirshman, W.I. van Rij and P. Merkel, Comput. Phys. Commun. 43, 143 (1986).

[12] A.H. Glasser, J.M. Greene and J.L. Johnson, Phys. Fluids. 19, 567 (1976).

[13] S. Okamura et al., Nucl. Fusion 39, 1337 (1999).

[14] S. Sakakibara et al., Nucl. Fusion 41, 1177 (2001).

[15] M. Wakatani et al., Fusion Eng. Des. 15, 395 (1992).

[16] S. Sakakibara et al., Fusion Sci. Tech. 50, 177 (2006). 